\title{
Relationship among nocturnal sleep deficit, excess weight and metabolic alterations in adolescents
}

\author{
Nelina Ruiz, M.D..$^{a, b}$, Airam Rangel, M.D. ${ }^{b}$, Carla Rodríguez, M.D. ${ }^{b}$, \\ Lisette Rodríguez, M.D. ${ }^{b}$, and Valeria Rodríguez, M.D. ${ }^{b}$
}

\begin{abstract}
Introduction. Sleep modulates neuroendocrine function and metabolism; therefore, changes in sleep duration may lead to developing obesity during adolescence.

Objective. To assess the possible association among nocturnal sleep duration, the presence of overweight and metabolic alterations in a group of adolescents.

Population and Methods. Cross-sectional, analytical study conducted at a school in Valencia, Venezuela, during the 2012-2013 school year. Participants were 12 to 17 year-old adolescents. A survey on nocturnal sleep duration was administered; weight, height and waist circumference were recorded; and glycemia, lipid profile and insulinemia levels were measured. Body mass index and the homeostasis model assessment of insulin resistance (HOMA-IR) index were calculated.
\end{abstract}

Results. Ninety adolescents were included. Compared to the group with normal weight, adolescents with excessive weight had, in average, fewer sleep hours Sundays through Thursdays $(p<0.05)$ and a higher rate of sleep deficit and sleep debt $(p<0.05)$. Low HDL cholesterol and insulin resistance was significantly associated with sleep $\operatorname{debt}(p<0.05)$. Among adolescents with sleep debt, the risk of having excess weight was 2.70 times higher (95\% CI $=1.09-6.72 ; p=0.032)$ regardless of age, gender, sexual maturity, sleep deficit Sundays through Thursdays, and history of cardiovascular disease and diabetes in first-degree relatives. Conclusions. Nocturnal sleep deficit and sleep debt were significantly associated with excess weight and metabolic alterations related to a high cardiometabolic risk.

Key words: adolescence, sleep, childhood obesity, insulin resistance.

http:/ /dx.doi.org/10.5546/aap.2014.eng.511

\section{INTRODUCTION}

Adolescents gain greater autonomy and independence in their eating and sleeping habits and are therefore more vulnerable to overnutrition and sleep disorders. The National Venezuelan Institute of Nutrition reported a $12.03 \%$ of overweight in adolescents (13-17 years old), while obesity affected $9.33 \%$ of youth in the 2008-2010 period. ${ }^{1}$ In addition, nocturnal sleep insufficiency ( $<8 \mathrm{~h} /$ day) is also prevalent among adolescents. ${ }^{2,3}$

Sleep is a restorative process that modulates neuroendocrine function and metabolism. ${ }^{4}$

For this reason, reduced sleep duration may lead to metabolic and hormonal deregulation, thus favoring food intake, ${ }^{5}$ obesity and cardiometabolic risk. A systematic review and a meta-analysis of studies conducted on adolescents indicated a reverse association between sleep duration and obesity, although some studies have not disclosed such finding in young women. ${ }^{6}$

The expression "sleep debt" is widely used to describe the effects associated with sleep deprivation, notwithstanding its causes, and is defined as the accumulation of lost sleep hours in relation to daily sleep requirements. ${ }^{7}$ Along with normal maturational changes, adolescents tend to delay their bedtime, and this combined with starting their academic and social activities even earlier results in a sleep deprivation pattern that is usually compensated on weekends. ${ }^{8}$

Although North American and European studies have revealed a relationship between sleep and obesity in adolescents, ${ }^{9,10}$ there are no data available in Venezuela. Considering that socio-cultural and environmental conditions that impact on the habits of adolescents may vary among countries, we proposed to assess nocturnal sleep duration and debt and any possible association with obesity and metabolic alterations 
in a sample of adolescents from the district of Valencia, Venezuela.

\section{POPULATION AND METHODS}

This was a field study with an analyticalcorrelational, cross-sectional, non-experimental design using a purposive, non-probability sampling. The study protocol was approved by the Ethics Committee of Universidad de Carabobo.

The study population was made up of adolescents (12-17 years old) enrolled at a private school in the district of Valencia, State of Carabobo, Venezuela, during the 20122013 school year. The purpose of the investigators was to assess all the above-mentioned population; however, the estimated required sample size to meet the study objective was calculated considering obesity prevalence in Venezuelan youth $(9 \%),{ }^{1}$ a $95 \%$ confidence level, and a $5 \%$ accuracy. The resulting sample size was 89 adolescents.

A written notice was provided to the eligible population to invite them to participate, which explained the study objectives and the research protocol. Adolescents and parents and/or their representatives were asked to sign the informed consent/assent at the same time. Inclusion criteria were having signed the informed consent/assent and attending the scheduled assessments. Exclusion criteria were as follows: body mass index (BMI) with a $\mathrm{Z}$ score below -2 as per the World Health Organization (WHO) 2007 growth reference standard, ${ }^{11}$ personal history of cardiovascular disease, uncontrolled blood hypertension (HTN), cancer diagnosis, diabetes mellitus (DM), kidney or liver failure, any chronic neurological, autoimmune or inflammatory disease (rheumatoid arthritis or ankylosing spondylitis), any thyroid or adrenal disease, recent major trauma or surgery, insulin, corticosteroid or psychotropic therapy, participation in a weight loss program, and employment activity with alternating day and/ or night shifts.

A survey was administered to adolescents and parents to collect personal data, medical history of HTN, DM, transient ischemic attack (TIA), stroke both in participants and their firstdegree relatives. Adolescents retrospectively indicated, by recalling, the time they went to bed and woke up each day of the week. Based on this information, we were able to establish sleep duration during the week and on weekends. In this study, Fridays and Saturdays were considered weekend days. The following indicators were estimated: SS-T: average sleep hours on a weekday (Sundays through Thursdays), SF-S: average sleep hours on a weekend day (Fridays and Saturdays), WS: overall week weighted sleep hours, calculated as follows: [(average number of sleep hours during the week $x$ 5) + (average number of sleep hours during the weekend $x 2$ )]/7. Sleep deficit and sleep excess were defined, respectively, when indicators were below or above the $10^{\text {th }}$ and $90^{\text {th }}$ percentiles of previously reported nocturnal sleep duration for their age. ${ }^{12}$

There is no uniformity in the literature regarding how sleep debt is calculated. As Leger, et al., ${ }^{13}$ we assumed that individuals compensated sleep lost during the week by sleeping in on weekends, so we estimated the difference in sleep hours between both periods (SF-S - SS-T). If such difference was $\geq 2 \mathrm{~h}$, it was defined as sleep debt (SD).

Weight and height were measured using a previously calibrated mechanical scale (Health o meter) (accuracy $=0.1 \mathrm{~g}$ and $0.1 \mathrm{~cm}$, respectively), with adolescents on a standing position and with their heads adjusted to the Frankfurt plane and shoulders relaxed so as to prevent lordosis. ${ }^{14}$ Adolescents were barefoot, wearing their school uniform but no socks, accessories and/or jewelry. BMI $\left(\mathrm{kg} / \mathrm{m}^{2}\right)$ and $\mathrm{Z}$ scores for BMI were estimated using the AnthroPlus software, version 1.02, based on the WHO 2007 child growth reference standard and the BMI cut-off points recommended by the WHO. ${ }^{11}$

With subjects standing, their waist circumference (WC) was measured using a tape measure positioned at the midline between the last rib and the iliac crest at the end of an unforced expiration. The presence of abdominal obesity was established based on the Venezuelan pediatric reference for WC. ${ }^{15}$ Sexual maturity status was established by self-assessment of pubertal development by looking at standard photographs of Tanner stages..$^{16,17}$

A 10-mL blood sample was collected following a 12-14 hour fasting and having eaten a light meal at dinner. Serum glucose, total cholesterol (TC), HDL cholesterol (HDL-C) and triglycerides (TGL) were determined using enzymatic-colorimetric methods. LDL cholesterol (LDL-C) was determined according to Friedewald formula. The following cardiovascular risk ratios were estimated: TC/HDL-C, LDL-C/ 
HDL-C, TGL/HDL-C, and non-HDL cholesterol (TC-HDL-C). Based on serum glucose and insulin levels measured using ELISA, the homeostasis model assessment of insulin resistance (HOMA-IR) index was obtained. ${ }^{18}$

DM was defined as glycemia $\geq 126 \mathrm{mg} /$ dL. ${ }^{19}$ TC, LDL-C, HDL-C, TGL and non-HDL cholesterol alterations were defined as per the cut-off points recommended by the National Heart, Lung and Blood Institute (NHLBI) for adolescents ${ }^{20}$ while recommendations for adults were used for TC/HDL-C and LDL-C/HDL-C ratios. ${ }^{21} \mathrm{~A}$ TGL/HDL-C $\geq 2.0$ was considered elevated. ${ }^{22}$

Based on gender and pubertal maturity stages, insulinemia and the HOMA-IR index were considered high if they were $\geq 90^{\text {th }}$ percentile reported by Barja, et al..$^{23}$

Central tendency and absolute and relative frequencies were estimated as descriptive statistics. The Kolmogorov-Smirnov test was used to check if outcome measures followed a normal distribution. Student unpaired $t$ tests or Mann-Whitney $\mathrm{U}$ tests were used to compare sleep indicators, as applicable, by age and anthropometric nutritional status. Chi-square tests and Fisher's exact tests were used to assess the possible association among sleep duration alterations, excess weight and metabolic alterations. A logistic regression analysis allowed to establish if excess weight or abdominal obesity, coded as present $=1$ or absent $=0$, have been predicted by the presence of $\mathrm{SD}$, regardless of age, gender, sexual maturity, sleep deficit Sundays through Thursdays and history of HTN, TIA, stroke and DM in first-degree relatives. A forward stepwise selection was used for introducing/ removing outcome measures into/from the logistic regression model. The significance level was estimated at $p<0.05$. Analyses were performed using the PASW Statistics software, version 18.0 for Windows.

\section{RESULTS}

One hundred and seven adolescents signed the consent/assent form and attended the scheduled assessment. Once inclusion/exclusion criteria were applied, the sample was made up of 90 adolescents (Figure 1). The group's average age was $14.8 \pm 1.4$ years old, $58.9 \%(n=53)$ were aged 15 to 17 years old and $41.1 \%(n=37), 12$ to 14 years old. Most participants were girls (72.2\%, $n=65$ ). The relative frequency of first-degree relatives with a history of HTN, TIA, stroke and DM was $30 \%, 5.6 \%, 1.1 \%$ and $5.6 \%$, respectively.
Table 1 shows anthropometric and metabolic indicators assessed by gender, with expected anthropometric differences.

SF-S, WS and the difference of sleep hours between weekends and weekdays (SF-S - SS-T) were significantly higher in adolescents aged 12 to 14 years old compared to 15 to 17 year-olds (Table 2). No significant differences by gender were observed in relation to the analyzed sleep indicators.

Table 3 shows the frequency of sleep duration alterations in the overall sample and by age, pointing out that $42.2 \%$ of participants had sleep deficit on SS-T; $53.3 \%$ had SD; and 61.1\% slept in excess on Fridays and Saturdays. Sleep deficit on SS-T $(\mathrm{OR}=0.295,95 \% \mathrm{CI}=0.122-0.711$; $p=0.005)$ and $\mathrm{SD}(\mathrm{OR}=0.368,95 \% \mathrm{CI}=0.153$ $0.884 ; p=0.020)$ were significantly more common among 12 to 14 year-old adolescents than among 15 to 17 year-old ones. The frequency of sleep duration alterations, including excess sleep, was not associated with gender (data not shown).

In terms of BMI, $21.1 \%$ of participants $(n=19)$ were overweight and $14.4 \%(n=13)$ were obese; $42.2 \%(n=38)$ had abdominal obesity as per their WC. Given the low number of obesity cases, sleep duration and debt were compared by BMI and studied individuals were grouped into two new categories: normal weight and excess weight, and the latter included overweight and obese participants (Table 4). SS-T was observed to be significantly lower in adolescents with excess weight when compared tonormal weight ones; similarly, WS tended to be lower $(\mathrm{p}=0.053)$. The same analysis performed by grouping individuals by WC showed that the difference in sleep hours between weekends and weekdays (SF-S - SS-T) was significantly higher in adolescents with abdominal obesity than in those with a normal WC $(2.49 \pm 1.72$ versus $1.73 \pm 1.77$ hours; $p=0.046$ ); the remaining sleep indicators showed no variation in terms of WC (data not shown).

Rates of sleep deficit S-T $(\mathrm{OR}=2.443$, $95 \% \mathrm{CI}=1.009-5,911 ; p=0.038)$, sleep deficit F-S $(\mathrm{OR}=3.284,95 \% \mathrm{CI}=1.232-8.757 ; p=0.016)$ and $\mathrm{SD}(\mathrm{OR}=2.708,95 \% \mathrm{CI}=1.091-6.721 ; p=0.025)$ in adolescents with excess weight were significantly higher compared to those who had a normal BMI (Table 5). The presence of abdominal obesity was also significantly associated with $\mathrm{SD}(\mathrm{OR}=3.632$, $95 \% \mathrm{CI}=1.488-8.863 ; p=0.004)$. Neither excess weight nor abdominal obesity were significantly associated with excessive sleep duration.

Sleep deficit S-T was associated with insulin resistance $(\mathrm{OR}=3.348,95 \% \mathrm{CI}=1.095-10.231$; 
FIGURE 1. Flowchart of study sample selection

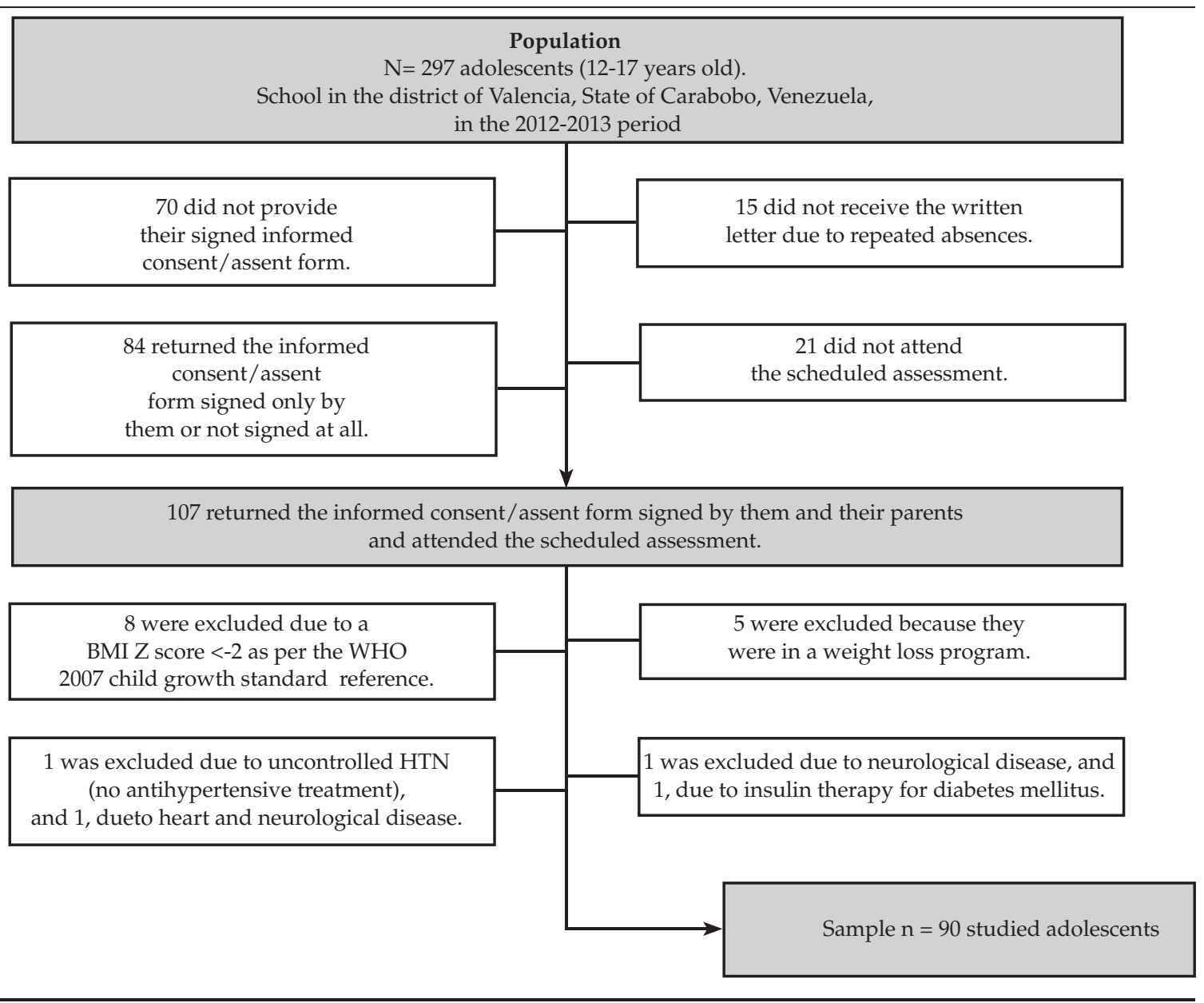

TABLE 1. Anthropometric and metabolic indicators assessed for the overall sample and by gender

\begin{tabular}{lcccc}
\hline Outcome measures & Overall group $(\mathbf{n}=\mathbf{9 0})$ & Males $(\mathbf{n}=\mathbf{2 5} ; \mathbf{2 7 . 8 \%})$ & Females $(\mathbf{n}=\mathbf{6 5} ; \mathbf{7 2 . 2} \mathbf{\%})$ & $\mathbf{p}$ \\
\hline Weight $(\mathrm{kg})$ & $58.2 \pm 14.3$ & $63.9 \pm 13.7$ & $56.0 \pm 14.1$ & 0.008 \\
Height (m) & $1.60 \pm 0.08$ & $1.67 \pm 0.09$ & $1.57 \pm 0.07$ & 0.000 \\
BMI (kg/m2) & $22.7 \pm 4.5$ & $22.8 \pm 4.1$ & $22.6 \pm 4.7$ & 0.893 \\
BMI Z score & $0.62 \pm 1.16$ & $0.82 \pm 1.07$ & $0.55 \pm 1.20$ & 0.336 \\
WC (cm) & $73.1 \pm 11.5$ & $76.1 \pm 10.8$ & $72.0 \pm 11.7$ & 0.043 \\
Glycemia (mg/dL) & $85.3 \pm 5.6$ & $87.2 \pm 5.5$ & $84.6 \pm 5.9$ & 0.052 \\
TGL (mg/dL) & $68.3 \pm 35.6$ & $60.4 \pm 34.0$ & $71.3 \pm 35.9$ & 0.193 \\
TC (mg/dL) & $141.7 \pm 29.3$ & $137.6 \pm 31.1$ & $143.3 \pm 28.6$ & 0.404 \\
HDL-C (mg/dL) & $33.3 \pm 6.3$ & $32.6 \pm 5.4$ & $33.5 \pm 6.6$ & 0.567 \\
LDL-C (mg/dL) & $94.7 \pm 25.2$ & $92.8 \pm 25.7$ & $95.5 \pm 25.2$ & 0.647 \\
TC/HDL-C ratio & $4.34 \pm 0.98$ & $4.24 \pm 0.85$ & $4.39 \pm 1.03$ & 0.527 \\
LDL-C/HDL-C ratio & $2.91 \pm 0.86$ & $2.86 \pm 0.80$ & $2.93 \pm 0.90$ & 0.716 \\
TGL/HDL-C ratio & $2.16 \pm 1.32$ & $1.89 \pm 1.06$ & $2.26 \pm 1.40$ & 0.238 \\
Non-HDL TC (mg/dL) & $108.4 \pm 27.5$ & $104.8 \pm 28.6$ & $109.8 \pm 27.2$ & 0.450 \\
Insulin (mIU/mL) & $12.5 \pm 5.6$ & $11.2 \pm 5.6$ & $12.9 \pm 5.5$ & 0.226 \\
HOMA-IR index & $2.63 \pm 1.19$ & $2.40 \pm 1.23$ & $2.71 \pm 1.18$ & 0.318 \\
\hline
\end{tabular}

Results expressed as arithmetic mean \pm standard deviation. Student $\mathrm{t}$ test or Mann-Whitney $\mathrm{U}$ test, as applicable.

BMI: body mass index; WC: waist circumference; TGL: triglycerides; TC: total cholesterol;

HDL-C: high-density lipoprotein cholesterol; LDL-C: low-density lipoprotein cholesterol. 
$p=0.027)$ and hyperinsulinemia $(\mathrm{OR}=5.4$, $95 \% \mathrm{CI}=1.338-21.791 ; p=0.013)$; the six adolescents with hyperinsulinemia and the four adolescents with insulin resistance had sleep deficit S-T. In addition, the number of cases with low HDL-C $(\mathrm{OR}=3.903,95 \% \mathrm{CI}=1.137-13.397 ; p=0.023)$ and with insulin resistance $(\mathrm{OR}=3.854,95 \% \mathrm{CI}=1.002-$ 15.232; $p=0.041$ ) was significantly higher among adolescents with SD. None of the studied metabolic alterations was associated with excessive sleep duration $(p \geq 0.05)$.

The risk of excess weight in adolescents with SD was 2.70 times higher $(95 \% \mathrm{CI}=1.09$ -
6.72; $p=0.032$ ) when compared to those with no SD. Similarly, the risk of having abdominal obesity was 3.92 times higher $(95 \% \mathrm{CI}=1.54-10.03$; $p=0.004$ ) in adolescents with SD versus those with no SD, regardless of age, gender, sexual maturity, presence of sleep deficit S-T and history of TIA, stroke and DM in first-degree relatives, although this was not the case with family history of HTN $(95 \% \mathrm{CI}=1.40-10.02 ; p=0.011)$.

\section{DISCUSSION}

In this study, WS hours for the overall sample were $8.37 \mathrm{~h} /$ day, similar to what has been

TABLE 2. Nocturnal sleep indicators for the overall sample and by age

\begin{tabular}{|c|c|c|c|c|}
\hline \multirow{2}{*}{ Sleep indicators } & Overall sample $(n=90)$ & \multicolumn{2}{|c|}{ Age group } & \multirow[t]{2}{*}{$\mathbf{p}$} \\
\hline & & $12-14$ years old $(n=37)$ & $15-17$ years old $(n=53)$ & \\
\hline Sleep Sundays through Thursdays $\left(\mathrm{S}_{\mathrm{S}-\mathrm{T}^{\prime}}\right.$ hours $)$ & $7.78 \pm 1.11$ & $7.99 \pm 1.11$ & $7.63 \pm 1.10$ & 0.138 \\
\hline Sleep Fridays and Saturdays ( $\mathrm{S}_{\mathrm{F}-\mathrm{S}^{\prime}}$ hours) & $9.86 \pm 1.95$ & $10.59 \pm 1.76$ & $9.36 \pm 1.94$ & 0.003 \\
\hline Week weighted sleep (WS, hours) & $8.37 \pm 1.10$ & $8.73 \pm 1.04$ & $8.13 \pm 1.08$ & 0.009 \\
\hline $\begin{array}{l}\text { Difference between sleep hours } \\
\text { on weekends and weekdays }\left(\mathrm{S}_{\mathrm{F}-\mathrm{S}}-\mathrm{S}_{\mathrm{S}-\mathrm{T}} \text { hours }\right)\end{array}$ & $2.08 \pm 1.94$ & $2.60 \pm 1.82$ & $1.72 \pm 1.95$ & 0.034 \\
\hline
\end{tabular}

Results expressed as arithmetic mean \pm standard deviation. Unpaired Student $\mathrm{t}$ test.

TABLE 3. Nocturnal sleep duration alterations for the overall sample and by age group

\begin{tabular}{|c|c|c|c|c|c|}
\hline \multirow{2}{*}{$\begin{array}{l}\text { Nocturnal sleep } \\
\text { duration alterations }\end{array}$} & \multirow{2}{*}{$\begin{array}{c}\text { Overall sample } \\
\mathrm{n}(\%) \\
\end{array}$} & \multicolumn{2}{|c|}{ Age group } & \multirow{2}{*}{$\begin{array}{c}\text { Fisher's } \\
\text { exact test }\end{array}$} & \multirow[b]{2}{*}{$\mathrm{p}$} \\
\hline & & 12-14 years old $n(\%)^{*}$ & 15-17 years old $n(\%)^{*}$ & & \\
\hline Sleep deficit S-T & $38(42.2)$ & $22(59.5)$ & $16(30.2)$ & 0.009 & 0.005 \\
\hline Sleep deficit F-S & $10(11.1)$ & $4(10.8)$ & $6(11.3)$ & 1.000 & 0.610 \\
\hline Weekly sleep deficit & $23(25.6)$ & $13(35.1)$ & $10(18.9)$ & 0.092 & 0.068 \\
\hline Sleep debt & $48(53.3)$ & $25(67.6)$ & $23(43.4)$ & 0.032 & 0.020 \\
\hline Excess sleep S-T & $6(6.7)$ & $1(2.7)$ & $5(9.4)$ & 0.394 & 0.207 \\
\hline Excess sleep F-S & $55(61.1)$ & $25(67.6)$ & $30(56.6)$ & 0.380 & 0.204 \\
\hline Weekly excess sleep & $16(17.8)$ & $4(10.8)$ & $12(22.6)$ & 0.173 & 0.121 \\
\hline
\end{tabular}

overall $n=90 ; 12-14$ year-old group $n=37 ; 15-17$ year-old group $n=53$.

* Percentages were estimated based on the total number of subjects in each age group.

S-T: Sundays through Thursdays; F-S: Fridays and Saturdays.

TABLE 4. Nocturnal sleep indicators by body mass index category

\begin{tabular}{|c|c|c|c|}
\hline Sleep indicators & Normal weight(n = 58) & Excess weight $(n=32)$ & $\mathbf{p}$ \\
\hline Sleep Sundays through Thursdays $\left(\mathrm{S}_{\mathrm{S}-\mathrm{T}^{\prime}}\right.$ hours) & $7.98 \pm 1.04$ & $7.42 \pm 1.15$ & 0.021 \\
\hline Sleep Fridays and Saturdays ( $\mathrm{S}_{\mathrm{F}-\mathrm{S}}$, hours) & $9.95 \pm 1.95$ & $9.72 \pm 1.99$ & 0.596 \\
\hline Week weighted sleep (WS, hours) & $8.54 \pm 1.04$ & $8.08 \pm 1.14$ & 0.053 \\
\hline $\begin{array}{l}\text { Difference between sleep hours on } \\
\text { weekends and weekdays }\left(\mathrm{S}_{\mathrm{F}-\mathrm{S}}-\mathrm{S}_{\mathrm{S}-\mathrm{T}^{\prime}} \text { hours }\right)\end{array}$ & $1.96 \pm 1.94$ & $2.30 \pm 1.95$ & 0.440 \\
\hline
\end{tabular}

Results expressed as arithmetic mean \pm standard deviation. Unpaired Student $\mathrm{t}$ test. 
observed in Spanish adolescents $(8.35 \mathrm{~h} / \text { day })^{24}$ and in adolescents from ten different European cities $\left(8 \mathrm{~h} /\right.$ day).${ }^{9}$ However, breaking down results by weekday, the average number of sleep hours $\mathrm{S}-\mathrm{T}$ was $7.78 \mathrm{~h} /$ day versus $9.86 \mathrm{~h} /$ day on F-S, and these figures are lower than those reported in a sample of French adolescents $(8.43 \mathrm{~h}$ /day and $10.01 \mathrm{~h} /$ day, respectively).$^{13}$ In addition, and although it is not easy to make comparisons given the dissimilar definitions used by authors for sleep deficit and debt, this research revealed that sleep deficit rates S-T and SD are higher than those reported by other studies on European adolescents. ${ }^{9,13}$

The difference in sleep hours between weekends and weekdays (SF-S - SS-T) was significantly higher among 12-14 year-old adolescents when compared to the 15-17 yearold group. Besides, cases of sleep deficit S-T and SD were significantly more frequent in the younger adolescents (12-14 years old), although this group had a greater average of sleep hours F-S, probably because they try to compensate lost hours of sleep on weekdays by sleeping in on weekends; however, in many cases, such compensation was not enough. Such observations disagree with those made by other authors, who have described a reduction in sleep duration among Saudi adolescents as their age increased, with no significant differences by gender, ${ }^{2}$ and a higher SD among 15-year old French adolescents when compared to 11-year old ones. ${ }^{13}$ Such disagreement may be accounted for by differences in the socio-cultural and socio-economic contexts. In addition, it could be pointed out that during the early stages of adolescence, there is probably a greater tendency to devote leisure time to technology-related activities and spend nights playing videogames and using social networks. It is also probable that, at present, parents tend to pay less attention to the number of hours their children spend doing such activities at night. On their side, older and more independent groups, tend to spend their leisure time interacting with the opposite sex and going out every week at night, especially on Fridays and Saturdays. This is probably reflected on the lower average number of hours of sleep F-S in 15-17 year-old adolescents.

In this study, sleep deficit was significantly associated with excess weight, although that was not the case of excess sleep, and this confirms the results of a meta-analysis conducted by Chen, et al., ${ }^{6}$ and of other recent cross-sectional ${ }^{9}$ and longitudinal ${ }^{10,25}$ studies. Regardless of age, gender, sexual maturity, presence of sleep deficit S-T and history of TIA, stroke and DM in first-degree relatives, the risk of excess weight was almost three times higher in adolescents with SD. Likewise, the risk of abdominal obesity was almost four times higher, which is consistent with the fact that sleep duration is a risk factor for excess weight and visceral fat accumulation in adolescents. In line with the above, it has been demonstrated that a longer sleep duration at the beginning of a three-month weight management program could predict the reduction of one $\mathrm{kg} / \mathrm{m}^{2}$ or more at the end of it. ${ }^{26}$

The presence of SD was associated with low HDL-C and insulin resistance levels, which is consistent with the observations made by other authors. ${ }^{27,28}$ The following are the biological and behavioral mechanisms proposed as an explanation for weight gain and metabolic alterations induced by a short sleep duration: increased appetite due

TABLE 5. Nocturnal sleep duration alterations by body mass index category

\begin{tabular}{lcccc}
\hline $\begin{array}{l}\text { Nocturnal sleep } \\
\text { duration alterations }\end{array}$ & Normal weight $n(\%)$ & Excess weight $n(\%)$ & Fisher's exact test & p \\
\hline Sleep deficit S-T & $20(34.5)$ & $18(56.3)$ & 0.074 & 0.038 \\
Sleep deficit F-S & $5(8.6)$ & $5(15.6)$ & 0.319 & 0.250 \\
Weekly sleep deficit & $10(17.2)$ & $13(40.2)$ & 0.222 & 0.016 \\
Sleep debt & $26(44.8)$ & $22(68.8)$ & 0.046 & 0.025 \\
Excess sleep S-T & $6(10.3)$ & $0(0)$ & 0.085 & 0.065 \\
Excess sleep F-S & $38(65.5)$ & $17(53.1)$ & 0.267 & 0.176 \\
Weekly excess sleep & $11(19.0)$ & $5(15.6)$ & 0.780 & 0.464 \\
\hline
\end{tabular}

overall $n=90$; normal weight group $n=58$; excess weight group $n=32$.

Percentages were estimated based on the total number of subjects in each body mass index category group.

S-T: Sundays through Thursdays; F-S: Fridays and Saturdays. 
to ghrelin hypersecretion (orexigenic hormone) and leptin hyposecretion (anorexigenic hormone), reduced energy expenditure, more chances of eating during the night, diets with a low nutritional quality, and reduced physical activity due to the fatigue induced by sustained alertness. ${ }^{5,29}$ An hyperactive sympathetic nervous system and hypercortisolemia may contribute to insulin resistance in association with sleep reduction. ${ }^{5}$

It is recommended that health and educational entities develop educational campaigns aimed at adolescents and parents on how sleep helps to maintain a good cardiometabolic health status. In addition, new longitudinal studies should be conducted with larger sample sizes and including other outcome measures (socio-economic stratum, diet and recreational activities).

Lastly, this research poses certain limitations given its cross-sectional design and because causative and temporal relations among assessed outcome measures cannot be confirmed. Besides, the sample was made up of individuals from a single school so it cannot be taken as representative of adolescents from other schools, cities or socio-economic strata. In addition, sleep duration in this study was self-reported instead of being recorded through polysomnographies (the gold standard); therefore, sleep duration may have been under-reported. Confounding outcome measures, such as physical activity in the studied population, were not assessed, so it is not possible to establish their impact on our findings.

\section{CONCLUSIONS}

In our sample of adolescents from the district of Valencia, Venezuela, nocturnal sleep deficit and sleep debt were significantly associated with excess weight and the presence of metabolic alterations related to a high cardiometabolic risk.

\section{REFERENCES}

1. Instituto Nacional de Nutrición. Sobrepeso y obesidad en Venezuela (prevalencia y factores condicionantes). Evaluación antropométrica. Caracas: FondoEditorialGente de Maíz; 2011:48-57.

2. Al-Hazzaa HM, Musaiger AO, Abahussain NA, AlSobayel HI, et al. Prevalence of short sleep duration and its association with obesity among adolescents 15- to 19year olds: A cross-sectional study from three major cities in Saudi Arabia. Ann Thorac Med 2012; 7(3):133-9.

3. McKnight-Eily LR, Eaton DK, Lowry R, Croft JB, et al. Relationships between hours of sleep and health-risk behaviors in US adolescent students. Prev Med 2011;53(45):271-3.

4. Beccuti G, Pannain S. Sleep and obesity. Curr Opin Clin
Nutr Metab Care 2011;14(4):402-12.

5. Lucassen EA, Rother KI, Cizza G. Interacting epidemics? Sleep curtailment, insulin resistance, and obesity. Ann N Y Acad Sci 2012;1264:110-34.

6. Chen X, Beydoun MA, Wang Y. Is sleep duration associated with childhood obesity? A systematic review and metaanalysis. Obesity (Silver Spring) 2008;16(2):265-74.

7. Van Dongen HP, Rogers NL, Dinges DF. Sleep debt: Theoretical and empirical issues. Sleep Biol Rhythms 2003;1(1):5-13.

8. Sánchez-Carpintero Abad R. Trastornos del sueño en la niñez. En Asociación Española de Pediatría, Sociedad Española de Neurología Pediátrica, eds. Protocolos de Neurología. 2. da ed. Madrid: Asociación Española de Pediatría;2008:255-61.

9. Garaulet M, Ortega FB, Ruiz JR, Rey-López JP, et al. Short sleep duration is associated with increased obesity markers in European adolescents: effect of physical activity and dietary habits. The HELENA study. Int J Obes (Lond) 2011;35(10):1308-17.

10. MitchellJA, RodriguezD,SchmitzKH,Audrain-McGovern J. Sleep duration and adolescent obesity. Pediatrics 2013;131(5):e1428-34.

11. De Onis M, Onyango AW, Borghi E, Siyam A, et al. Development of a WHO growth reference for school-aged children and adolescents. Bull World Health Organ 2007; 85(9):660-7.

12. Iglowstein I, Jenni OG, Molinari L, Largo RH. Sleep duration from infancy to adolescence: reference values and generational trends. Pediatrics 2003;111(2):302-7.

13. Leger D, Beck F, Richard JB, Godeau E. Total sleep time severely drops during adolescence. PLoS One 2012;7(10):e45204.

14. Izaguirre de Espinoza I, López de Blanco M. Evaluación del crecimiento y de la maduración física. En Sociedad Venezolana de Pediatría y Puericultura, ed. Nutrición Pediátrica. Caracas: Panamericana; 2009:1-40.

15. Hidalgo G, Flores-Torres J, Rodríguez-Morales A, Vásquez E, et al. Determinación de puntos de corte para la circunferencia de cintura a través de curvas ROC en población pediátrica de tres regiones de Venezuela evaluada en el SENACREDH. Arch Venez Pueric Pediatr 2011;74(3):95-9.

16. Marshall WA, Tanner JM. Variations in pattern of pubertal changes in girls. Arch Dis Child 1969;44(235):291-303.

17. Marshall WA, Tanner JM. Variations in the pattern of pubertal changes in boys. Arch Dis Child 1970;45(239):13-23.

18. Matthews DR, Hosker JP, Rudenski AS, Naylor BA, et al. Homeostasis model assessment: insulin resistance and beta-cell function from fasting plasma glucose and insulin concentrations in man. Diabetologia 1985;28(7):412-9.

19. AmericanDiabetes Association. Diagnosisand classification of diabetes mellitus. Diabetes Care 2010;33 Suppl 1:S62-9.

20. National Institute of Health. National Heart, Lung and Blood Institute. Expert Panel on Integrated Guidelines for Cardiovascular Health and Risk Reduction in children and adolescents. Full Report. NIH Publication N ${ }^{\circ} 12-7486$. Bethesda: National Institute of Health; 2012.

21. Millán J, Pintó X, Muñoz A, Zúñiga M, et al. Lipoprotein ratios: Physiological significance and clinical usefulness in cardiovascular prevention. Vasc Health Risk Manag 2009;5:757-65.

22. Di Bonito P, Moio N, Scilla C, Cavuto L, et al. Usefulness of the high triglyceride-to-HDL cholesterol ratio to identify cardiometabolic risk factors and preclinical signs of organ damage in outpatient children. Diabetes Care 2012;35(1):158-62.

23. Barja S, Arnaiz P, Domínguez A, Villarroel L, et al. Insulinemia e índice HOMA en niños y adolescentes 
chilenos. Rev Med Chile 2011;139(11):1435-43.

24. Ortega FB, RuizJR, Castillo R, Chillón P, etal.Sleep duration and cognitive performance in adolescence. The AVENA study. Acta Paediatr 2010;99(3):454-6.

25. Seegers V, Petit D, Falissard B, Vitaro F, et al. Short sleep duration and body mass index: a prospective longitudinal study in preadolescence. Am J Epidemiol 2011;173(6):621-9.

26. Sallinen BJ, Hassan F, Olszewski A, Maupin A, et 1. Longer weekly sleep duration predicts greater 3 month BMI reduction among obese adolescents attending a clinical multidisciplinary weight management program. Obes Facts 2013;6(3):239-46
27. JavaheriS, Storfer-Isser A, Rosen CL, RedlineS. Association of short and long sleep durations with insulin sensitivity in adolescents. J Pediatr 2011;158(4):617-23.

28. Azadbakht L, Kelishadi R, Khodarahmi M, Qorbani M, et al. The association of sleep duration and cardiometabolic risk factors in a national sample of children and adolescents: the CASPIAN III study. Nutrition 2013;29(9):1133-41.

29. TaheriS. The link between short sleep duration and obesity: we should recommend more sleep to prevent obesity. Arch Dis Child 2006;91(11):881-4. 